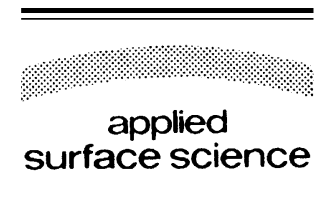

Applied Surface Science 214 (2003) 109-119

www.elsevier.com/locate/apsusc

\title{
Self-assembly of micro- and nano-scale particles using bio-inspired events
}

\author{
H. McNally ${ }^{\mathrm{a}}$, M. Pingle ${ }^{\mathrm{b}}$, S.W. Lee ${ }^{\mathrm{a}}$, D. Guo ${ }^{\mathrm{a}}$, D.E. Bergstrom ${ }^{\mathrm{b}}$, R. Bashir ${ }^{\mathrm{a}, \mathrm{c}, *}$ \\ ${ }^{\text {a }}$ School of Electrical and Computer Engineering, Purdue University, W. Lafayette, IN 47907, USA \\ ${ }^{\mathrm{b}}$ Department of Medicinal Chemistry, Purdue University, W. Lafayette, IN 47907, USA \\ ${ }^{\mathrm{c}}$ Department of Biomedical Engineering, Purdue University, W. Lafayette, IN 47907, USA
}

Received 27 June 2002; received in revised form 22 February 2003; accepted 1 March 2003

\begin{abstract}
High sensitivity chemical and biological detection techniques and the development of future electronic systems can greatly benefit from self-assembly processes and techniques. We have approached this challenge using biologically inspired events such as the hybridization of single (ss)- to double-stranded (ds) DNA and the strong affinity between the protein avidin and its associated Vitamin, biotin. Using these molecules, micro-scale polystyrene beads and nano-scale gold particles were assembled with high efficiency on gold patterns and the procedures used for these processes were optimized. The DNA and avidin-biotin complex was also used to demonstrate the attachment of micro-scale silicon islands to each other in a fluid. This work also provides insight into the techniques for the self-assembly of heterogeneous materials.
\end{abstract}

(C) 2003 Elsevier Science B.V. All rights reserved.

Keywords: Bio-inspired self-assembly; Nanoparticles; Heterogeneous integration

\section{Introduction}

While engineers and scientist are aspiring to controllably manipulate structures at the micro- and nanometer scale, nature has been performing these tasks and assembling complex structures with great accuracy and high efficiency using highly specific molecules such as DNA and proteins. Since Mirkin et al. [1] and Aliviastos et al. [2] demonstrated DNA mediated-assembly of gold nanoparticles; there has been a tremendous interest and many reports in the use of DNA to specifically assemble

\footnotetext{
* Corresponding author. Tel.: +1-765-496-6229; fax: +1-765-494-6441.

E-mail addresses: bergstrom@purdue.edu (D.E. Bergstrom), bashir@ecn.purdue.edu (R. Bashir).

URL: http://dynamo.ecn.purdue.edu/ bashir/.
}

micro- and nano-size particles for chemical and biological detection and for possible uses in electronic circuitry to assemble future nano-electronic devices. Numerous approaches to the challenge of assembly have been demonstrated to include biological entity mediated [3,4], chemically mediated [5], electrically mediated [6], and fluidics mediated [7]. Patterned assembly of micron-sized Au disk using electrostatic forces has been demonstrated [8]. However, these were not active devices, rather passive Au pads that were not suitable for any electronic applications. Active devices such as carbon nanotubes [9] and InGaAs LEDs [10] as well as metallic nanorods [11] for interconnect systems have been assembled. Additional work is required to bring these systems to application.

Nature assembles nano-scale components using molecular recognition. In the case of DNA, hydrogen 
bonding provides the specificity behind the matching of complementary pairs of single-stranded (ss) DNA to hybridize into a double strand (ds) of helical DNA. It has been estimated that each base pair binds with $0.5 \mathrm{kcal} / \mathrm{mol}$ of energy [12]. For the use of a $18 \mathrm{mer}$ oligonucleotide, the binding energy can be estimated at $9 \mathrm{kcal} / \mathrm{mol}$. The actual binding energy of a dsDNA is dependent on the base-pair sequence, salt concentration of surrounding media, temperature, among others. In the case of antibodies/antigens and ligands/receptors, binding takes places by a combination of electrostatic forces, chemical bonding, and shape-mediated effects. As an example, avidin is a large protein that has binding sites for four biotin molecules. The affinity of the biotin-avidin complex is equivalent to $21 \mathrm{kcal} / \mathrm{mol}$ [13]. In comparison, the $\mathrm{Au}-\mathrm{S}$ thiolate covalent bond, which is used to attach thiol conjugated oligonucleotides to gold surfaces has an energy of $44 \mathrm{kcal} / \mathrm{mol}$ [14]. Although, taken individually, these energies are considerably less than that for many covalent single bonds (e.g. the $\mathrm{C}-\mathrm{O}$ bond is $96 \mathrm{kcal} / \mathrm{mol}$ ), in composite, they are sufficiently strong to provide stable attachment at ambient temperatures.

In this study, the assembly of micro- and nano-scale particles using biologically inspired events such as DNA hybridization and ligand/receptor interactions has been investigated. Different approaches can be taken to particle assembly, as shown in Fig. 1(a-c). For all three cases, the particles could be beads, devices, or other nano and micro-scale objects. Fig. 1(a) presents an approach that uses DNA only and relies on hybridization for attachment of the islands to the surface [15]. The substrate surface is functionalized with ssDNA while the complementary strand is attached to the gold surface of the particle. Direct hybridization into a dsDNA would result in capture of the islands onto the patterned gold surface. Indirect hybridization has also been demonstrated, in which a third DNA strand is used to hybridize with the strands attached to the surface and to the particles. We have begun with the approach shown in Fig. 1(b), which utilizes DNA and ligand/receptors for the capture of avidin-coated particles. A dsDNA is attached to a surface presenting a biotin. The strong affinity between avidin and biotin is relied upon to ultimately capture the particle. This design allows for the verification of ssDNA attachment to the surface, hybridization into the dsDNA and the availability of biotin for particle capture. Fig. 1(c) also shows the capture of particles with a thin layer of gold so the particles may also be functionalized with DNA via the $\mathrm{Au}-\mathrm{S}$ bond. The ssDNA is attached to the gold surface and subsequently hybridized just as the patterned surface is functionalized. Again, the dsDNA present biotin molecules for attachment to the avidin molecule. Avidin is first absorbed on the substrate surface and then the particles are captured using one of the other three sites of the immobilized avidin. We have used

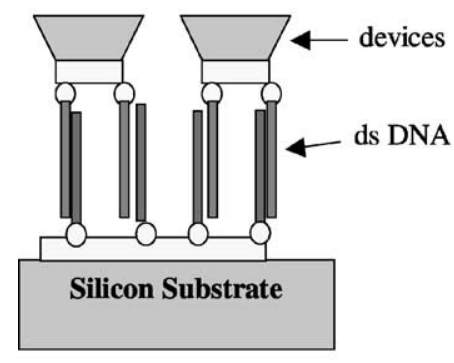

(a)

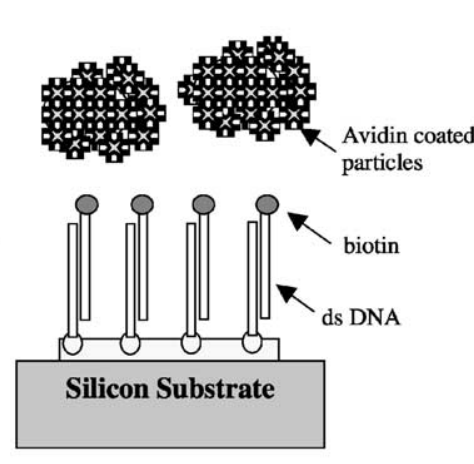

(b)

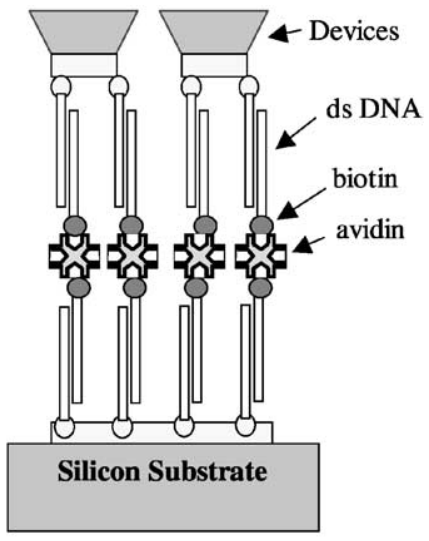

(c)

Fig. 1. Various strategies for bio-inspired assembly of particles (not drawn to scale). (a) DNA on both devices and substrates, (b) use of biotinylated-DNA on substrate and avidin on particles, (c) use of biotinylated-dsDNA on both substrate and devices and avidin as linking element. 
the approach depicted in Fig. 1(c) for the attachment of devices to each other in a fluid.

\section{Materials and procedures}

Variations in the biological entities and labeling, and the sequence of surface and particle functionalization were used during experimentation which are described below.

\subsection{Biological entities}

Biological events such as DNA hybridization and protein interactions were used for the assembly of micro- and nano-scale particles. 18mer oligonucleotides were obtained through the Laboratory for Macromolecular Structure at Purdue University. Variations have been used for differing attachment schemes and labeling requirements. These are presented in Table 1, along with the function of each sequence. The thiolated oligonucleotides were used for attachment to gold surfaces, either on patterned silicon substrates or on the particles to be assembled. The same oligonucleotides without thiol molecules were used as control for attachment experiments. Non-complementary hybridization sequences were used as control for hybridization. FITC and rhodamine labeling was also used for attachment and hybridization verification.

The oligonucleotides presenting a biotin at the $5^{\prime}$-end were used when the experiment involved the biotin-avidin interaction for particle assembly. Biotin (Glen Research, Cat. No. 10-5950-95) was attached to the $5^{\prime}$-end during the DNA synthesis. Avidin (Pierce, Cat. No. 21121) was used for absorption to biotin previously attached to patterned surfaces or to particles for assembly.

\subsection{Functionalization and verification}

The patterned surfaces used for particle assembly were prepared in-house using standard silicon processing. A silicon oxide layer was grown and a photoresist patterned developed. Chrome and gold layers of 300 and $500 \AA$, respectively were deposited, the chrome providing an adhesion layer. The substrates were cleaved into $3 \mathrm{~mm} \times 3 \mathrm{~mm}$ pieces for experimental samples. Prior to use, each samples was cleaned in a UV ozone clean system (Jelight, Model 342) and covered with phosphine $\left(\mathrm{C}_{6} \mathrm{H}_{5} \mathrm{P}\left(\mathrm{C}_{6} \mathrm{H}_{4} \mathrm{SO}_{3} \mathrm{~K}\right)_{2} \mathrm{P} \cdot 2 \mathrm{H}_{2} \mathrm{O}\right.$ from Stream Chemicals, Cat. No. 15-0463) for $12 \mathrm{~h}$. The use of phosphine was adapted from gold nanoparticle experiments [16] in which the authors showed that the phosphine passivated the surface until it was displaced by a thiol, allowing the $\mathrm{Au}-\mathrm{S}$ covalent bond to occur. The substrates were then rinsed lightly in DI, dried with a nitrogen flow and placed in a clean vial for DNA application, with all chemical processing done in nitrogen atmosphere.

Our procedure for ssDNA surface attachment and hybridization was adapted from the process to attach DNA strands to gold nanoparticles [17]. Briefly, attachment begins by placing $300 \mu \mathrm{l}$ of $15 \mu \mathrm{M}$ thiolated ssDNA in water on the cleaned samples surface. The solution and sample is allowed to incubate on a shaker for $12 \mathrm{~h}$. Then $75 \mu \mathrm{l}$ of a $5 \times$ phosphate buffer is added to the vials and the solution is again allowed to incubate on a shaker for $24 \mathrm{~h}$. The sample is then carefully rinsed in $0.3 \mathrm{M}$ phosphate-saline buffer. At this point the attachment can be verified if fluorescently labeled ss attachment sequence is used

Table 1

Oligonucleotides used for the DNA-based assembly experiments

\begin{tabular}{lll}
\hline Sequence \# & Function & Sequence \\
\hline 1 & Attachment & $5^{\prime}-($ SH)-C-A-G-T-C-A-G-G-C-A-G-T-C-A-G-T-C-A-3' \\
2 & Attachment w/FITC & $5^{\prime}-($ SH)-C-A-G-T-C-A-G-G-C-A-G-T-C-A-G-T-C-A-(F)-3' \\
3 & Control attachment no thiol w/FITC & $5^{\prime}-C-A-G-T-C-A-G-G-C-A-G-T-C-A-G-T-C-A-(F)-3^{\prime}$ \\
4 & Hybridization w/biotin \& FITC & $5^{\prime}-(B)-($ F)-T-G-A-C-T-G-A-C-T-G-C-C-T-G-A-C-T-G-3' \\
5 & Non-complementary Hyb w/biotin & $5^{\prime}-(B)-G-C-A-A-T-C-C-C-G-T-A-G-G-A-C-A-T-C-3^{\prime}$ \\
6 & Attachment w/biotin & $5^{\prime}-($ SH)-C-A-G-T-C-A-G-G-C-A-G-T-C-A-G-T-C-A-3'-(B) \\
7 & Control attachment (no thiol) w/biotin & $5^{\prime}$-C-A-G-T-C-A-G-G-C-A-G-T-C-A-G-T-C-A-3'-(B) \\
\hline
\end{tabular}

B: biotin, F: FITC. 


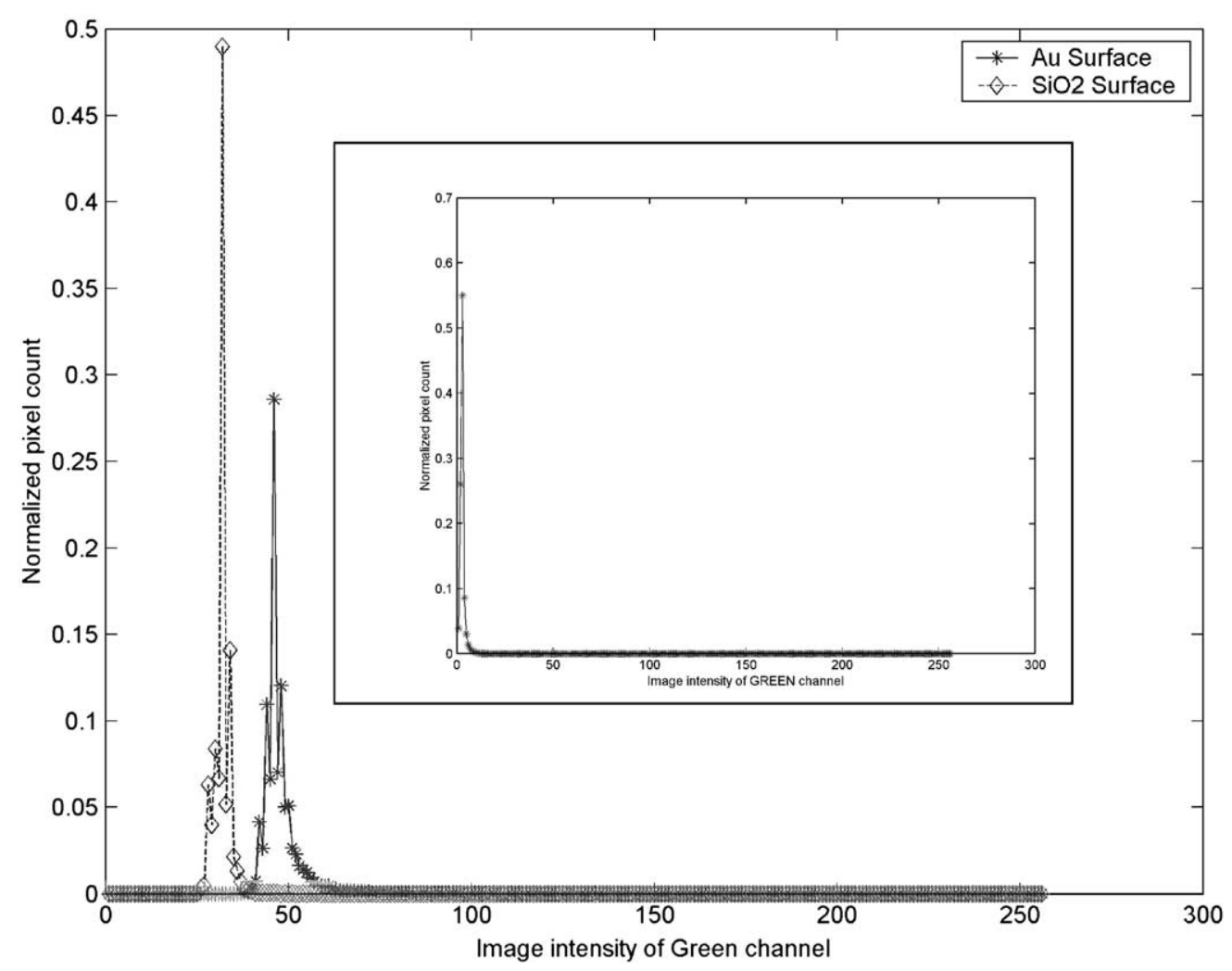

Fig. 2. Fluorescence verification of DNA attachment to patterned gold surfaces (using Sequence \#2, inset used Sequence \#3).

(Table 1). Hybridization is also performed on the same substrate. The substrate is placed into a cleaned vial with $15 \mu \mathrm{M}$ solution of the complementary ssDNA sequence in a phosphate and $\mathrm{NaCl}$ buffer. The vial is then placed in a water bath at $78{ }^{\circ} \mathrm{C}$ for $10 \mathrm{~min}$. The water bath with vials is allowed to cool slowly to room temperature and the samples are again rinsed in $0.3 \mathrm{M}$ phosphate-saline buffer. Our results show this attachment and hybridization procedure is successful on the patterned assembly substrates as well as the silicon particles (to be discussed in later sections).

Fluorescent tagging was used as an initial validation of the presence of the molecules on the gold surface after the attachment and hybridization steps. As shown in Fig. 2, an increase in fluorescence when using Sequence \#2, when compared to control samples (inset, with Sequence \#3) is clear, however, the fluorescence signal varied from sample to sample, possibly due to the quenching effects of the nearby metallic surface [18]. Consequently, fluorescence was only used for detection and not for quantification. Reflection-absorption infared spectroscopy (RAIRS) will be used in future studies to detect DNA attachment, orientation, and quantification.

\section{Experimental results and discussion}

\subsection{Polystyrene bead assembly and optimization}

The assembly of avidin-coated $0.8 \mu \mathrm{m}$ diameter polystyrene beads [19] onto patterned substrates was initially investigated due to the availability of various sizes and labels in addition to the high concentrations and low mass. The substrates functionalized with dsDNA presenting a biotin molecule were placed in a solution of avidin-coated beads and PBS. The avidin-biotin reaction was performed for $45 \mathrm{~min}$ at $37{ }^{\circ} \mathrm{C}$ while swirling the vial contents. Rinsing the sample under a stream of DI water was found to 


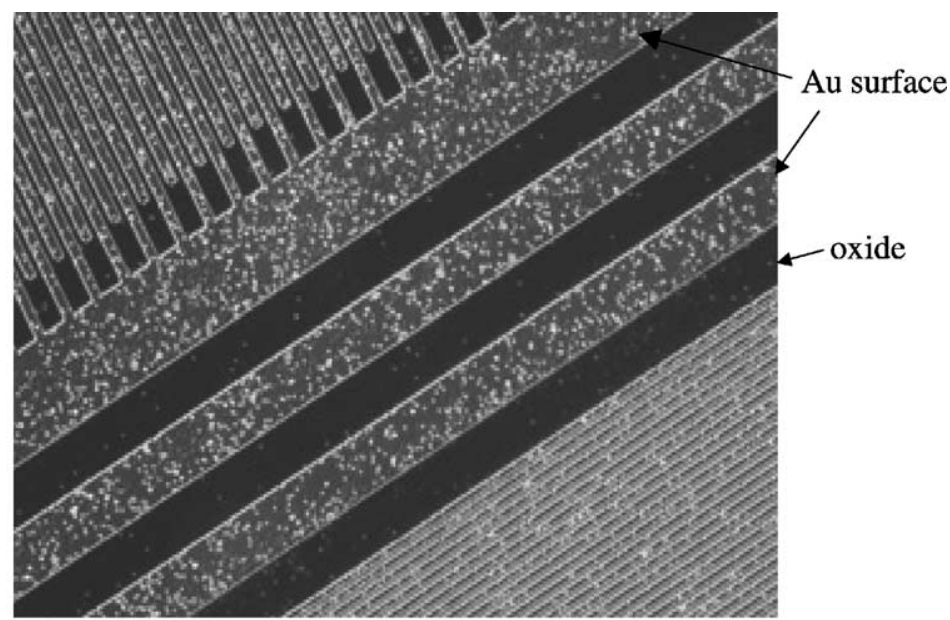

(a)

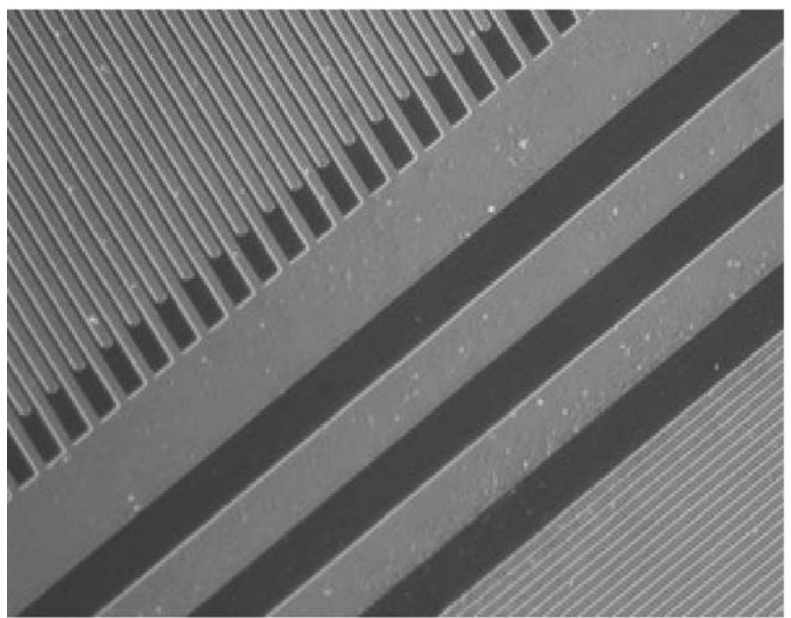

(b)

Fig. 3. Optical micrographs showing the capture of avidin-coated beads using biotinylated-DNA. (a) Sample showing the bead attachment on the gold surface (beads appear as dots), (b) control sample with no thiol, and hence no bead capture. Pictures taken at $400 \times$.

release the non-specifically bound beads while allowing the beads attached by the avidin-biotin bond to remain on the DNA treated gold surfaces. This procedure was also used for the assembly of gold nanoparticles [20].

In addition to the fluorescent verification discussed in the previous section, the confirmation of DNA oglionucleotide attachment to the surface (Sequence \#1) and subsequent hybridization (Sequence \#4) was verified using avidin-coated beads. If the oligonucleotides were available and presenting the biotin molecule, an avidin-coated particle would be captured. Fig. 3(a) shows this attachment of beads to a DNA treated patterned surface. A typical control samples is shown in Fig 3(b) for which the attachment sequence (Sequence \#3) did not have a thiol for initial attachment to the surface. A second set of controls was done for non-complementary hybridization (Sequence \#5), which provided similar results (i.e. no attachment). Such experiments can be used for detecting complementary sequences of DNA with very high sensitivity and very low detection limits.

Additional experiments were performed using polystyrene beads to optimize the attachment and hybridization procedures. Fig. 4 is a comparison of bead attachment on ssDNA versus a hybridized double 


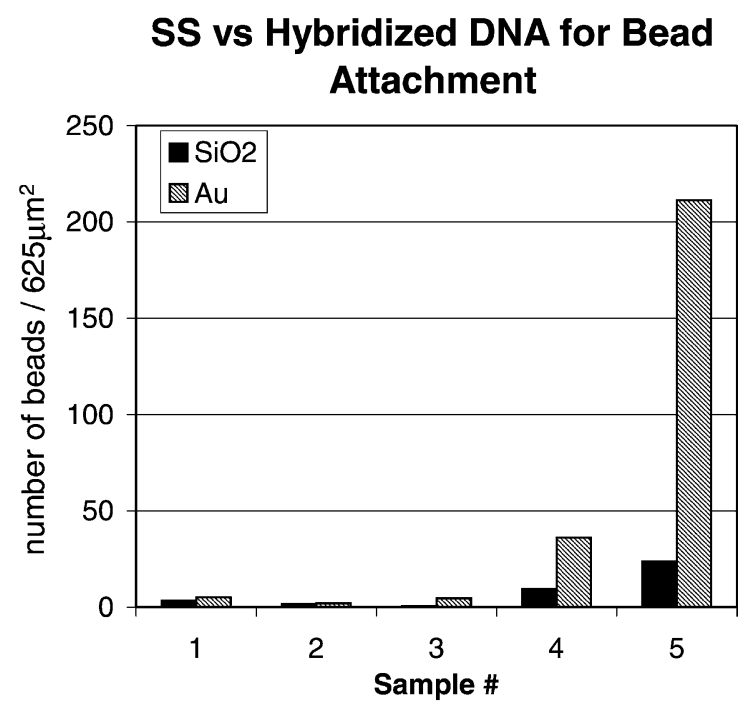

Fig. 4. ssDNA compared to hybridized dsDNA for bead capture. Sample 1 (Sequence \#6), sample 2 (Sequence \#7), sample 3 (Sequence \#3 \& \#4), sample 4 (Sequence\#1 \&\#5), sample 5 (Sequence\#1 \&\#4).
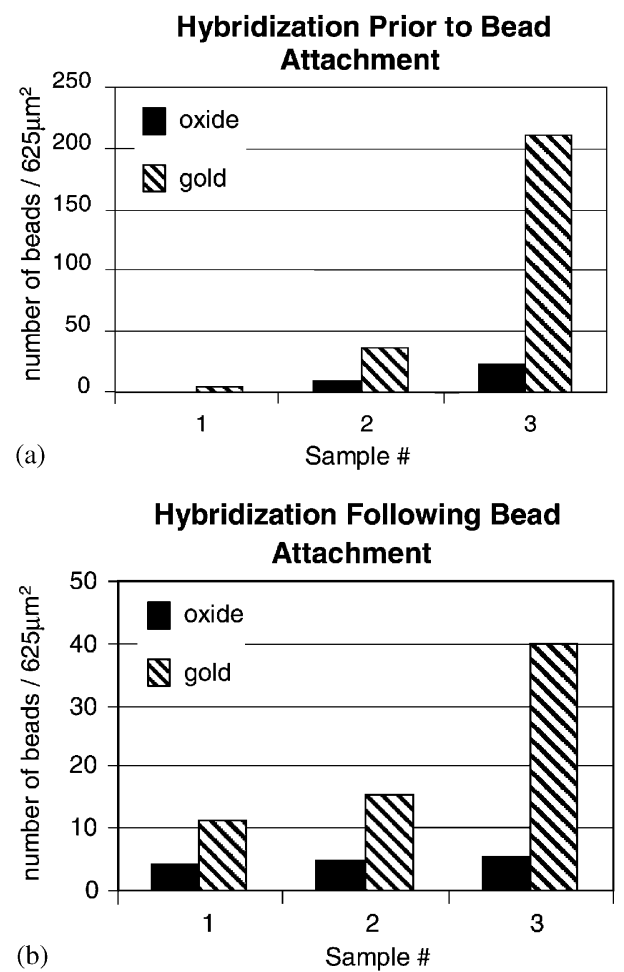

Fig. 5. Polystyrene bead capture with different attachment process sequences. (a) Hybridization prior to bead attachment, (b) hybridization following bead attachment. For both figures, sample 1 (Sequence \#3), sample 2, (Sequence \#1 \& \#5), sample 3 (Sequence \#1 \& \#4). strand. In both cases, a biotin molecule was presented to capture the avidin-coated beads. Samples 1 (Sequence \#6) and 2 (Sequence \#7) are both with ssDNA; sample 2 being the control in which the attachment sequence did not have a thiol for attachment to the gold surfaces. Samples 3 (Sequence \#3 and \#4), 4 (Sequence \#1 and \#5), and 5 (Sequence \#1 and \#4) are the hybridized versions. Samples 3 and 4 are the control samples, which included attachment control (no thiol) and non-complementary hybridization control, respectively. The surfaces presenting a biotin on a hybridized DNA strand clearly provided better attachment of beads than ssDNA. This may be due to the 'floppy' nature of a ssDNA on a surface [21]. The biotin may be too close to the substrate and not available to the beads for attachment.
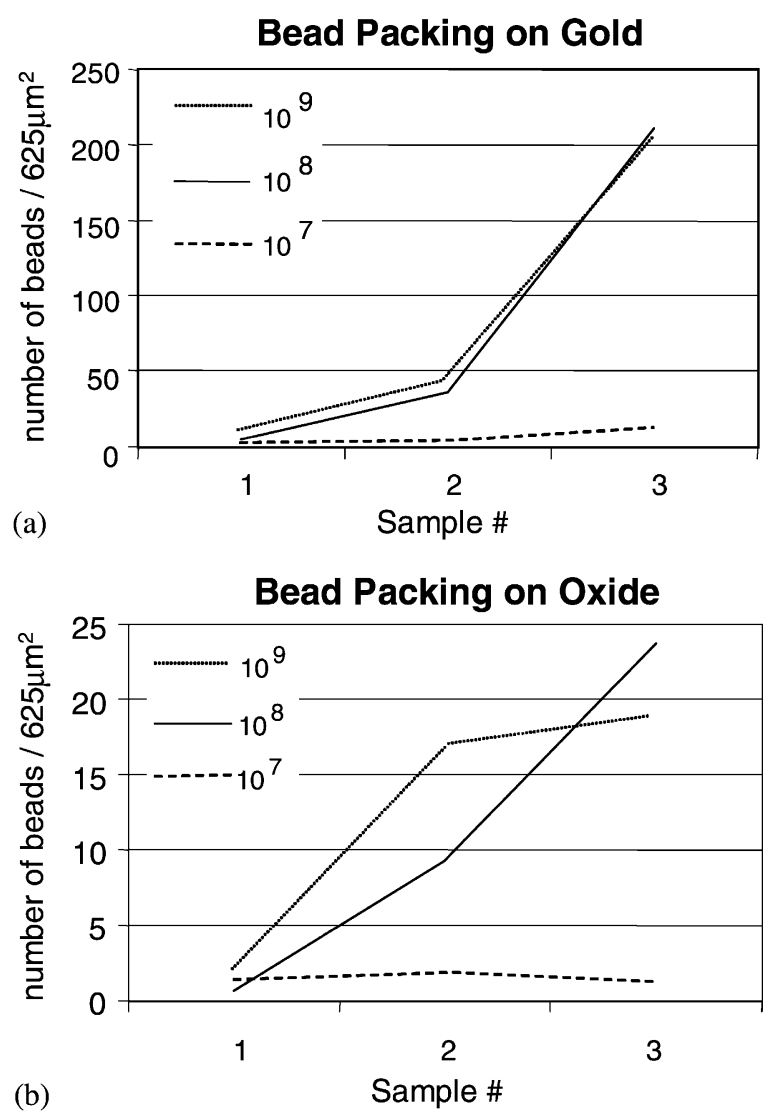

Fig. 6. Polystyrene bead capture with varying concentration of the beads. (a) On Au surface, (b) on $\mathrm{SiO}_{2}$ surface. For both figures, sample 1 (Sequence \#3), sample 2, (Sequence \#1 \& \#5), sample 3 (Sequence \#1 \& \#4). 
The sequence of attachment was investigated to optimize assembly. In one case the beads were attached after hybridization as described above. In the second method, the complementary sequence was attached to the avidin-coated beads via the biotin-avidin bond. Hybridization was then allowed to occur between the oligonucleotides thus assembling the attached bead. As shown in Fig. 5, the latter method resulted in less efficient bead attachment. Again, three samples are shown in each case: control attachment (no thiol, Sequence \#7), control hybridization (non-complementary, Sequence \#1, and \#5) and the hybridization sample

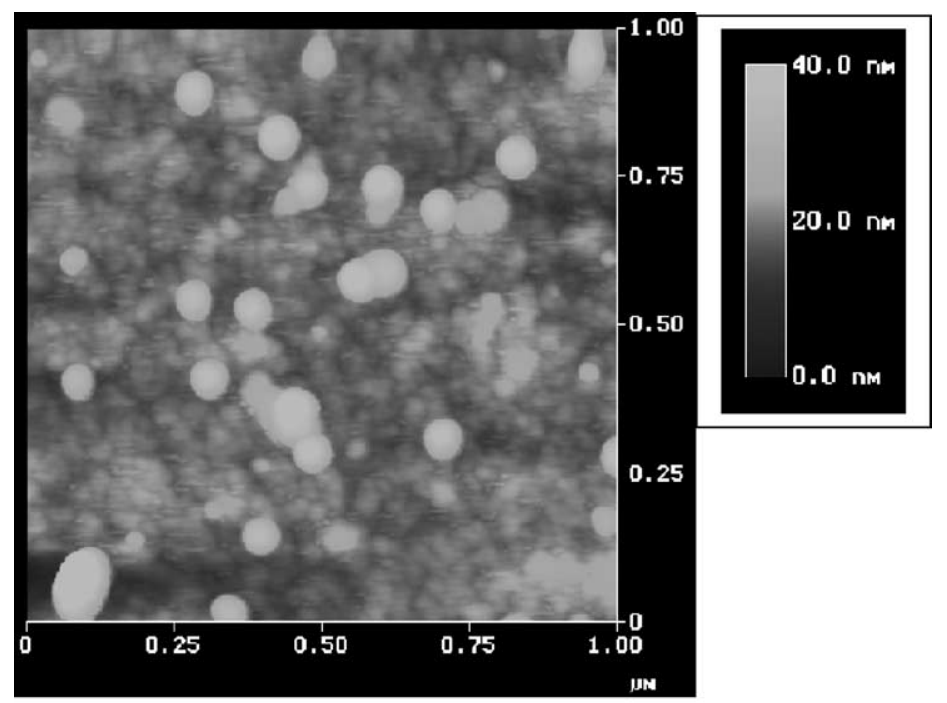

(a)

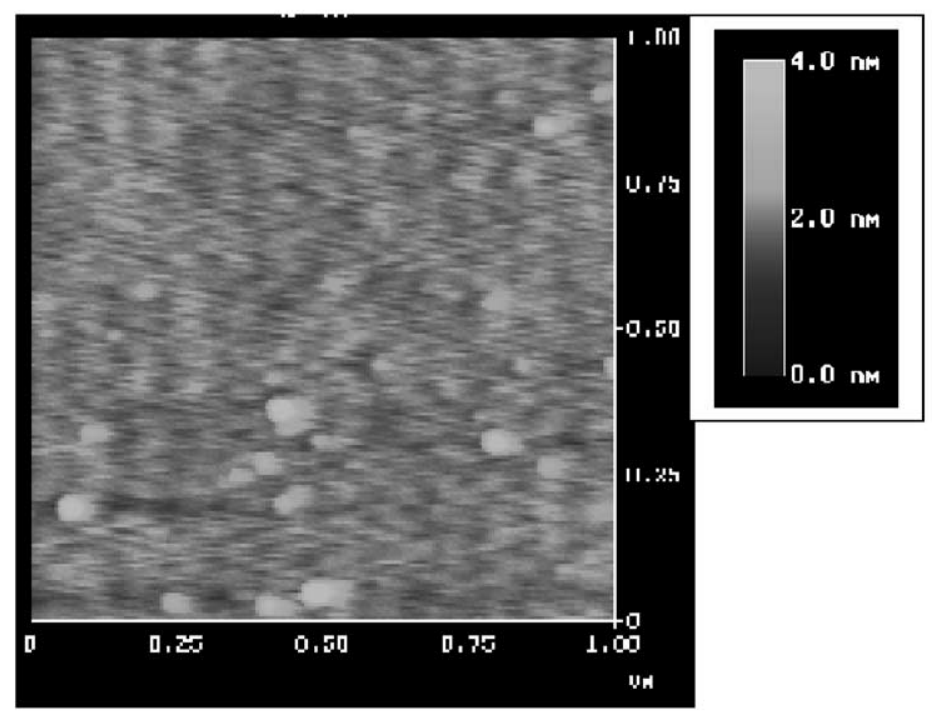

(b)

Fig. 7. Atomic force microscope scans of gold nanoparticle assembly on gold surface. (a) Nanoparticles captured on gold when DNA was attached to the substrate (Sequence \#1 \& \#5), (b) control sample with DNA which did not have thiols and hence no biotin was present to capture the streptavidin-coated nanoparticles (Sequence \#3 \& \#4). Note that the scale is different in the two figures. 
(Sequence \#1, and \#4). The attached bead could limit the complementary oligonucleotides from reaching the sequence on the substrate surface, thus reducing the efficiency of bead assembly.

The concentration of beads was also varied to investigate packing of the beads on the surface while minimizing the non-specific binding. Fig. 6 presents the results using three different concentrations of beads. Again, three cases are presented, which are the same as ones used in Fig. 5. The results show that concentrations of $10^{8}$ and $10^{9} \mathrm{ml}^{-1}$ provided similar attachment; however non-specific binding was higher with $10^{9} \mathrm{ml}^{-1}$ concentration. In addition, concentration of $10^{9} \mathrm{ml}^{-1}$ produced bead aggregates instead of individual bead assembly. A concentration of $10^{7} \mathrm{ml}^{-1}$ beads resulted in minimal bead assembly. Increased packing density of the beads may also be accomplished by increasing the DNA surface attachment density.

\subsection{Gold nanoparticle capture and assembly}

Reduction of particle size and increase in density are important for detection with high sensitivity and high spatial resolution. Attachment of nanoparticles to surfaces is of significant interest and has previously been accomplished [22,23]. In this work, we have assembled $20 \mathrm{~nm}$ gold nanoparticles onto gold patterns on a silicon substrate. The process is similar to the one described above for capture of avidin-coated polystyrene beads. Here, we have used streptavidincoated gold nanoparticles. The assembly substrate is functionalized with dsDNA with a biotin at the end of each molecule. This surface is then exposed to streptavidin-coated gold nanoparticles at a concentration of $10^{6} \mathrm{ml}^{-1}$ in PBS. The reaction was performed for $45 \mathrm{~min}$ at $37^{\circ} \mathrm{C}$ while swirling the vial contents. Removal of non-specifically bound nanoparticles was accomplished by placing the assembly substrate into PBS and sonication for $30 \mathrm{~s}$. The samples were then rinsed with DI water and dried with $\mathrm{N}_{2}$ prior to analysis. A Digital Instruments Dimension 3100 atomic force microscope (AFM) was used to obtain images of the surface in the tapping mode using an etched silicon tip (OTESPA). Fig. 7 shows an image of the nanoparticle assembly on gold (a) compared to the surrounding silicon oxide area (b). Fig. 8 provides quantification of the results. Control samples provided minimal nanoparticle attachment both on the silicon

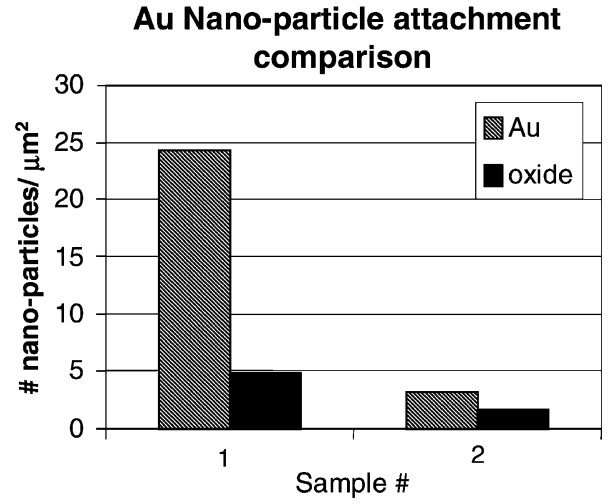

Fig. 8. Quantification of the capture for the gold nanoparticles on patterned gold and the oxide surface. Samples 1 (Sequence \#1\& \#5), and 2 (Sequence \#3 \& \#4).

oxide and gold surfaces. The assembled samples show minimal attachment on silicon oxide surface and increased attachment on the gold surface. Increased packing densities may be accomplished by further optimizing experimental conditions and by increasing the number of DNA molecules on the surface.

\subsection{Silicon islands assembly experiments and discussion}

Silicon particles as shown in Fig. 9, were fabricated as previously described [24] and used to demonstrate

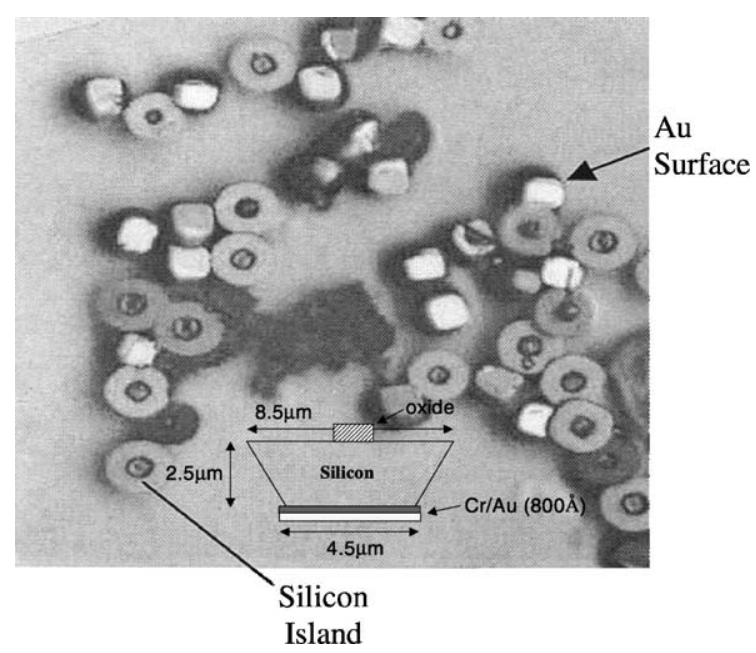

Fig. 9. Scanning Electron Micrograph of the released silicon islands using process described in [23]. Inset is a drawn crosssection of the silicon particle. 


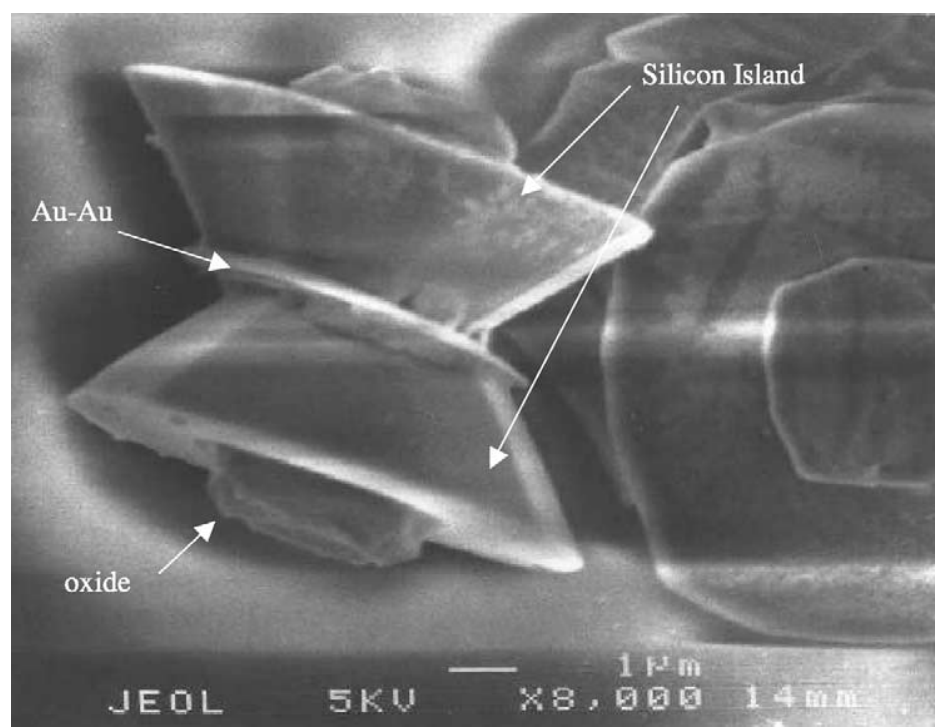

Fig. 10. Islands duplexes formed using the DNA and avidin-biotin interactions at the Au faces of the two islands.

possible integration of active electronic material. The silicon particles have a gold layer on one side to allow for molecular functionalization, which was performed prior to release from the handle substrate. Oligonucleotide attachment and hybridization of the particles attached to the handle substrate was performed as described earlier. The particles were then released into PBS using sonication and the handle substrate removed. Ninety-nine percent release of the particles has been accomplished consistently. The particles were collected using small centripetal forces to gently concentrate the particles, which could then be collected by pipetting. Concentrations of $10^{4}$ particles $/ \mathrm{ml}$ have been accomplished using this method, without damage to the particles. Assembly of the silicon particles requires additional processing. The particles were resuspended in a $1 \mathrm{mg} / \mathrm{ml}$ solution of avidin in PBS and heated to $37^{\circ} \mathrm{C}$ for $45 \mathrm{~min}$. The particles were then rinsed thoroughly and resuspended in water. The avidin bound to the biotin from two different particles and duplexes were formed with the gold sides facing each other as shown in Fig. 10. This was expected since the avidin molecule, which has four binding sites for biotin, would link biotins from two different particles, hence forming duplets. In a given experiment, $82 \%$ of the islands were found to be part of a duplex, whereas without the avidin in the solution, only $2 \%$ of the islands were found to be part of a duplex, most likely due to random non-specific interactions.

\section{Further discussion}

The assembly of micro- and nano-scale particles onto patterned surfaces using bio-inspired events is a significant challenge. Forces involved in assembly have included covalent, biological/molecular, electrostatic, gravity, and viscous drag. To complete the particle assembly for useful electronics, the substrates must be rinsed of non-specifically attached particles and dried. Thus, the forces of assembly must be greater than the forces encountered during subsequent processing. The combined biological events (DNA and protein layers) capture the assembled particles with estimated forces on the order of $10 \mathrm{kcal} / \mathrm{mol}$. A particle of surface area $16 \mu \mathrm{m}^{2}$, as shown in Fig. 9, would experience a maximum force of attachment of $10^{-7} \mathrm{~N}$ (or $10^{-12} \mathrm{~N}$ per molecule). This estimate of force includes optimal DNA attachment density of $10^{12}$ molecules/cm [11] and a hybridization efficiency of $30 \%$ [25]. Rinsing the substrates to remove nonspecifically bound particles is critical, specifically when the particle is large and dense as in the case of silicon particles coated with chrome and gold. The mass of the particle is $\sim 10^{-10} \mathrm{~g}$, yielding a force of gravity of $10^{-12} \mathrm{~N}$. The force of drag on the particles was estimated using Stoke's law for an equivalent volume of a sphere [26]. With a rinse velocity of $1 \mathrm{~cm} / \mathrm{s}$, the force of drag is $10^{-10} \mathrm{~N}$. Actual drag forces on a 
particle of this shape could be one order of magnitude higher. These estimated forces of particle attachment and release are highly dependent on our DNA attachment and hybridization efficiencies and the procedures used during substrate rinsing. Thus, forces experienced by the silicon particles during the rinsing and drying processes can be of roughly the same orders of magnitude as the binding forces for the current device size used.

Many alternatives are imagined to continue the assembly efforts. A flow through system may be designed in which the non-specific attachment is released with a flow velocity sufficient to remove non-specific attachments (due to gravity $10^{-12} \mathrm{~N}$ ) but reduce forces of drag $\left(10^{-9}\right.$ to $\left.10^{-10}\right)$ on the particles. Release of nonspecifically bound particles may also be accomplished as with the BARC sensor [23] but using electric fields. An electric field can be applied sufficient to attract the non-specifically bound particles, but not strong enough to break the biological bonds of those particles actually assembled. Work is also underway to reduce the goldcoated silicon particle size to $0.3 \mu \mathrm{m}^{3}$. This will reduce the force of gravity to $10^{-15} \mathrm{~N}$ and using the current rinsing procedures, the drag force to $10^{-12}$, comparable to that of the individual biological bonds. Finally, increased biological forces may be realized by: designing the oligonucleotide sequences for maximum hybridization forces, or altering the $\mathrm{pH}$ and salt contents of the surrounding media.

\section{Conclusions}

This paper has presented our progress towards the biologically inspired assembly of micro- and nanoscale particles. ssDNA attachment to patterned surfaces and the subsequent hybridization of a complementary strand was demonstrated. Polystyrene beads, with $0.8 \mu \mathrm{m}$ diameter and coated with avidin were captured and assembled on patterned gold surfaces functionalized with dsDNA with a biotin at the end. Streptavidin-coated gold nanoparticles were also captured using biotinylated-DNA on patterned surfaces. Silicon particles with gold on one side were used to form duplexes in a fluid due to binding of the DNA and avidin-biotin complex. Work is on going to perform the DNA and ligand/receptor-mediated self-assembly of silicon devices with reduced size. Additional work is required to bridge the span between current silicon processing and biological self-assembly. These techniques are expected to be applicable to the directed assembly of other nano-scale devices such as carbon nanotubes and silicon nano-wires.

\section{Acknowledgements}

This work was supported by Center of Nanoscale Devices at Purdue University funded through the State of Indiana, 21st Century Research and Technology Fund. We would also like to acknowledge Prof. Steve Wereley for helpful discussions and the Micro-fabrication facility and Staff at Purdue University for assistance in device processing.

\section{References}

[1] C.A. Mirkin, R.L. Letsinger, R.C. Mucic, J.J. Storhoff, Nature 382 (1996) 607.

[2] A.P. Alivisatos, K.P. Johnson, X. Peng, T.E. Wilson, C.J. Loweth, M.P. Bruchez, P.G. Schultz, Nature 382 (1996) 609.

[3] C.M. Niemeyer, B. Ceyhan, S. Gao, L. Chi, S. Peschel, U. Simon, Colloid Polym. Sci. 279 (2001) 68.

[4] R.L. Edelstein, C.R. Tamanaha, P.E. Sheehan, M.M. Miller, D.R. Baselt, L.J. Whitman, R.J. Colton, Biosens. Bioelectr. 14 (2000) 10.

[5] R.P. Andres, S. Datta, D.B. Janes, C.P. Kubiak, R. Reifenberger, The Handbook of Nanostructured Materials and Nano-Technology, Academic Press, New York, 1998.

[6] S.W. Lee, H.A. McNally, D. Guo, M. Pingle, D.E. Bergstrom, R. Bashir, Langmuir 18 (8) (2002) 3383-3386.

[7] H.J. Yeh, J.S. Smith, IEEE Photonics Technol. Lett. 6 (6) (1994) 706.

[8] J. Tien, A. Terfort, G.M. Whitesides, Langmuir 13 (1997) 5349.

[9] J. Liu, J. Casavant, M. Cox, D.A. Walters, P. Boul, W. Lu, A.J. Rimberg, K.A. Smith, D.T. Colbert, R.E. Smalley, Chem. Phys. Lett. 303 (1999) 125.

[10] C.F. Edman, R.B. Swint, C. Furtner, R.E. Formosa, S.D. Roh, K.E. Lee, P.D. Swanson, D.E. Ackley, J.J. Coleman, J.J. Heller, IEEE Photonics Technol. Lett. 12 (9) (2000) 1198.

[11] J.K.N. Mbindyo, B.D. Reiss, B.R. Martin, C.D. Keating, M.J. Natan, T.E. Mallouk, Adv. Mater. 13 (4) (2001) 249.

[12] E. Prohofsky, Statistical Mechanics and Stability of Macromolecules: Application to Bond Disruption, Base Pair Separation, Melting, and Drug Dissociation of the DNA Double Helix, Cambridge University Press, New York, NY, 1995.

[13] M. Wilcheck, E.A. Bayer (Eds.), Methods in Enzymology, vol. 184, 3 pp. 
[14] A. Ulman, Chem. Rev. 96 (4) (1996) 1533-1554.

[15] C.A. Mirkin, T.A. Taton, Nature 405 (8 June) (2000) 626-627.

[16] C.J. Loweth, W.B. Caldwell, X.G. Peng, A.P. Alivisatos, P.G. Schultz, Angew. Chem. Int. Ed. 38 (I12) (1999) 1808-1812.

[17] L.M. Demers, C.A. Mirkin, R.C. Mucic, R.A. Reynolds, R.L. Letsinger, R. Elghanian, G. Viswanadham, Anal. Chem. 72 (I22) (2000) 5535-5541.

[18] D.H. Waldeck, A.P. Alivastos, C.B. Harris, Surf. Sci. 158 (1985) 103-125.

[19] Spherotech Inc., Sphero Microparticles, Libertyville, IL.

[20] SPI Supplies, Structure Probe Inc., West Chester, PA.

[21] R. Levicky, T.M. Herne, M.J. Tarlov, S.K. Satija, J. Am. Chem. Soc. 120 (1998) 9787-9792.
[22] C.M. Niemeyer, B. Ceyhan, S. Gao, L. Chi, S. Peschel, U. Simon, Colloidal Polym. Sci. 279 (2001) 68-72.

[23] R.L. Edelstein, C.R. Tamanha, P.E. Sheehan, M.M. Miller, D.R. Baselt, L.J. Whitman, R.J. Colton, Biosens. Bioelectr. 14 (2000) 805-813.

[24] R. Bashir, S.W. Lee, D. Guo, M. Pingle, D. Bergstrom, H. McNally, D. Janes, Proceedings of the Materials Research Society, Fall 2000.

[25] L.M. Demers, C.A. Mirkin, R.C. Mucic, R.A. Reynolds, R.L. Letsinger, R. Elghanian, G. Viswanadham, Anal. Chem. 72 (22) (2000) 5535-5541.

[26] Frank M. White, Viscous Fluid Flow, second ed., McGraw Hill, Boston, MA, 1991. 